Canadian

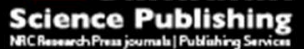

Canadian Journal of Civil Engineering Revue canadienne de génie civil

\title{
Experimental and computational study of a high-Reynolds jet flow
}

\begin{tabular}{|r|l|}
\hline Journal: & Canadian Journal of Civil Engineering \\
\hline Manuscript ID & cjce-2016-0433.R1 \\
\hline Manuscript Type: & Technical Note \\
\hline Date Submitted by the Author: & 24-Feb-2017 \\
\hline Complete List of Authors: & $\begin{array}{l}\text { Khosronejad, Ali; Stony Brook University, Civil Engineering } \\
\text { Feist, Christopher; University of Minnesota Twin Cities, St. Anthony Falls } \\
\text { Lab. } \\
\text { Marr, Jeff; University of Minnesota Twin Cities, St. Anthony Falls Lab. } \\
\text { Sotiropoulos, Fotis; Stony Brook University, Civil Engineering }\end{array}$ \\
\hline $\begin{array}{r}\text { Is the invited manuscript for } \\
\text { consideration in a Special } \\
\text { Issue? : }\end{array}$ & \\
\hline Keyword: & \\
\hline \multicolumn{2}{|c|}{} \\
\hline
\end{tabular}




\title{
Experimental and computational study of a high-Reynolds jet flow
}

\author{
Ali Khosronejad* ${ }^{1}$, Christopher Feist ${ }^{2}$, Jeff Marr ${ }^{3}$, and Fotis Sotiropoulos ${ }^{4}$
}

\begin{abstract}
Three-dimensional jet flows at high Reynolds $(R e)$ numbers, namely over a million, have a significant importance in hydraulic engineering. Despite their importance, most of previous investigations have been mainly focused only on jet flows with orders of magnitude lower Re numbers. We present the results of an experimental campaign and a high fidelity largeeddy simulation (LES) to study a jet flow with $R e \approx 1.7 \times 10^{6}$ in a large-scale flume. Flow measurements are carried out using a pitot tube apparatus and the Virtual Flow Simulator (VFSGeophysics) model is employed to simulate the flow field. The measured velocity field of the jet is used to evaluate the LES results. The presented experimental data for the cross-sectional velocity distributions at various distances from the jet source provide an unprecedented data-set for model validation at high $R e$ numbers.
\end{abstract}

Keywords: High Reynolds Jet, Large eddy simulation, Experimental study, Power spectra density.

\section{INTRODUCTION}

Jet flows have many applications in environmental fluid dynamics. The flow field downstream of many types of outlet apparatuses, e.g. outlet of power plants into marine or riverine stems, can be categorized as three-dimensional (3D) jets. Receiving environments for the 3D jets can be a stagnant or a moving body of water. One of the most crucial factors influencing the strength of the jet created shear layer and turbulence mixing mechanism is the disparity between the momentum of the jet and its receiving environment. A higher jet momentum means that its Reynolds $(R e)$ number will be higher, which in turn, leads to more complex flow field. The cost associated with either numerical or experimental studies of jet flows, on the other hand, can in fact increase with $R e$ number. Complexities involved in fully investigate the high $R e$ jet flows aside, it is important to quantify the effect of jet flows on their receiving environment in order to predict and remedy their unfavorable impacts on the geometry of

\footnotetext{
${ }^{1}$ Civil Engineering Dept., Stony Brook Univ., Stony Brook, USA, *Corresponding author, E-mail: ali.khosronejad@stonybrook.edu

${ }^{2}$ University of Minnesota, Minneapolis, USA

${ }^{3}$ University of Minnesota, Minneapolis, USA

${ }^{4}$ Civil Engineering Dept., Stony Brook Univ., Stony Brook, USA
} 
receiving water bodies, which among others, may include: natural rivers and streams, and artificial or natural water reservoirs.

Previous studies of 3D turbulent jets have been focused, among other subjects, on quantifying the velocity distribution and the rate at which the high velocity core decays downstream of the jet source. Such investigations were conducted using physical (Rajaratnam and Pani 1974; Craft and Launder 2001; Hall and D. 2007; Fellouah and Pollard 2009), analytical (Albertson et al. 1950; Rajaratnam and Subramanya 1967; Rajaratnam 1976), and numerical modeling (Wu and Rajaratnam 1995; Khosronejad and Rennie 2010). For instance, Wygnanski and Fiedlehr (1969), Rodi (1975), and Hussein et al. (1994) carried out detailed physical modelings to study axisymmetric jets flow with $R e$ numbers of about $10^{5}, 8.7 \times 10^{4}$, and $9.55 \times 10^{4}$, respectively. Capp et al. (1990), Panchapakesan and Lumley (1993) experimentally investigated the cascade of energy among various flow structures in jet flows with $R e$ numbers of about $10^{5}$, and $1.1 \times 10^{4}$, respectively. Mungal and Hollingsworth (1989) used image processing techniques to investigate the evolution of a laboratory jet flow ( $R e \approx 2 \times 10^{8}$ ). In another study by the same research team, Mungal et al. (1992) used the same techniques to investigate various scales of flow structures in a jet flow with $R e \approx 5 \times 10^{3}$ and compared their observations with those of $R e \approx 2 \times 10^{8}$. Furthermore, Yoda et al. (1992) experimentally studied far-field large-scale dynamics of their non-reacting $\left(R e \approx 2 \times 10^{8}\right)$ and a jet flame $\left(R e \approx 3 \times 10^{7}\right)$. (Kwon and Seo 2005) also studied the effect of $R e$ number on velocity distribution of jet flows with $R e<10^{4}$.

In the context of eddy-resolving numerical modeling, Bogey and Bailly (2009) and Bogey et al. (2012) used large-eddy simulation (LES) method to numerically investigate the evolution of the jet flows in the experiments of (Capp et al. 1990) and (Panchapakesan and Lumley 1993) in which $R e \approx 10^{5}$. (Chedevergne et al. 2015) have also recently studied both numerically and experimentally the evolution of round jets with the same $R e$ number.

Despite their great contributions to advance our knowledge of jet flows, past studies have been mainly focused on jet flows at low $R e$ (i.e., $R e<<10^{6}$ ). Only few have dealt with high $R e$ numbers. This state of affair can be attributed to the high cost associate with conducting experiment or high fidelity numerical simulations for jet flows at high Re numbers.

We herein report the results of a large-scale experimental campaign along with a high fidelity large-eddy simulation (LES) in which we attempt to investigate a confined, circular, water jet with a $R e$ number of $R e=U_{b} D / \nu=1.71 \times 10^{6}$, where $U_{b}$, $D$, and $\nu$ are the bulk velocity of the jet at its source, the diameter of the jet source, and the kinematic viscosity of water, respectively.

The experimental study shows that the jet's core velocity monotonically decays as the distance from the jet source increases. The main channel has rigid bed and side walls, however, during the experiment, at a distance of about $8 \mathrm{~m}$ downstream of the plane of the jet source there existed a frozen sand-pile. The frozen geometry of the sand pile is surveyed and included in the numerical simulation. We validated the LES results for the case with the frozen sand-pile.

We simulated the same jet flow in the main channel for two hypothetical cases: $(i)$ 
case A in which the sand pile was removed. This extra simulation is carried out to study the effect of the distal sand pile on the velocity distribution of the jet and (ii) case $\mathrm{B}$ in which we reduce the Re number by one order of magnitude to $1.71 \times 10^{5}$ (by reducing the bulk velocity to $1 / 10 t h$ of the experimental case). This simulation is carried out to compare the power spectral density (PSD) of the two jet flows. The details of the numerical method used in VFS-Geophysics model for solving the mean flow and turbulence closure governing equations have already been documented extensively elsewhere (Ge and Sotiropoulos 2007; Borazjani et al. 2008; Kang et al. 2011).

\section{EXPERIMENTAL SETUP}

The experimental study is carried out in a $1.83 \mathrm{~m}$ deep, $2.74 \mathrm{~m}$ wide, and $\approx 30 \mathrm{~m}$ long flume, which is supplied directly from Mississippi River through a $11.28 \mathrm{~m}$ long straight pipe with a diameter of $D=0.3048 \mathrm{~m}$. The jet's circular is approximately located in the middle of the main channel's cross sectional area, which in this work will be considered as the $y-z$ plane. Details of experimental setup and jet geometry are shown in Table 1 and Fig. 1. Downstream of the jet is a frozen sand pile with a frozen geometry that is illustrated in Table 2 . The sand pile geometry does not vary in $y$ direction, while its elevation $(z)$ varies with distance from the jet source $(x)$ until it becomes a flat surface at $x=10.36 \mathrm{~m}$.

To measure the streamwise flow velocity of the jet, we employed a pitot-static tube apparatus installed on an automated cart over the flume. The pitot-static tube probes the dynamic pressure using a Rosemount 3051S Coplanar Pressure Transmitter. The pressure transducer has a measurement range of 0 to $5 \mathrm{mH}_{2} \mathrm{O}$ with an accuracy of $0.025 \%$ over the span, i.e. $0.001 \mathrm{mH}_{2} \mathrm{O}$ in the present experiment. The diameter of the pitotstatic tube was $0.00635 \mathrm{~m}$ with a streamwise length of $0.1016 \mathrm{~m}$ and vertical height of $0.6096 \mathrm{~m}$. The pressure transducer outputs $4-20 \mathrm{~mA}$ over the differential pressure measurement range. A voltage was measured across a precision resistor, $200.2 \Omega$, by a Measurement Computing PCI-DAS4020/12 analog to digital card.

The streamwise flow velocities are measured (presented in the next section) on two planes: horizontal plane of $x-y$ and vertical plane of $x-z$. Coordinate of the center of the jet source is set as the origin where $x=y=z=0$. Measurements are carried out at various distances in: streamwise direction from $x=2 D$ to $10 D$ by $\Delta x=1 D$ apart; vertical direction from $z=-1.5 D$ below the jet source's center to $1.5 D$ above it by $\Delta z=0.25 D$; and spanwise direction from $y=-1.5 D$ on the left of the jet source to $1.5 D$ to the right of it by $\Delta y=0.25 D$.

\section{COMPUTATIONAL DETAILS}

The computational domains consist of the main channel rectangular flume in which the frozen sediment-pile, side walls, and the flume's rigid bed are embedded and treated as immersed bodies. The water supply pipe is simulated from the jet source to $5 \mathrm{~m}$ upstream of it. At the inlet of the supply pipe a previously saved, fully developed turbulent flow, is prescribed. The fully developed turbulent flow is obtained by running the model, in a separate simulation, for the flow within the supply tube with periodic boundary condition in the streamwise direction. Once the turbulence kinetic energy is 
statistically converged, the flow field at the output section of the pipe is saved for the jet flow simulation.

At the downstream end of the domain, which is located $2 m$ downstream of point 7 in Fig. 1 (see also Table 2), Neumann outlet boundary condition is employed for all three velocity components. The free surface boundary is treated as a rigid-lid while at the solid side walls and mobile bed the wall functions approach is employed (for more details see (Khosronejad and Sotiropoulos 2014)). The flume's rigid walls are treated as hydraulically smooth surfaces, while the effective roughness height of the frozen sand-pile is considered to be equal to $3 d_{50}$ of the sand material $\left(d_{50}=1.8 \mathrm{~mm}\right)$.

The computational grid size is determined based on a grid sensitivity analysis. The computational mesh consists of 47.8 million grid nodes that is $1201 \times 241 \times 165$ in streamwise $(x)$, spanwise $(y)$, and vertical $(z)$ directions, respectively. This grid arrangement implies that the grid resolution in $x, y$, and $z$ directions is $0.014001 \mathrm{~m}$, $0.011369 \mathrm{~m}$, and $0.011091 \mathrm{~m}$, respectively. Given the diameter of the jet source that is $0.3048 \mathrm{~m}$, with this grid resolution we resolve the jet source's area with about 27 grid points in both $y$ and $z$ directions. The non-dimensional time step $\left(\Delta t^{*}\right)$ is set equal to 0.01 , where the non-dimensional time is defined as $t^{*}=t U_{b} / D . \Delta t^{*}=0.01$ is chosen such that the maximum CourantFriedrichsLewy (CFL) number is less than 1.0.

The simulation is carried out using 160 processors on a Linux cluster composed of $2320 \mathrm{CPU}$ of AMD Opteron $2.6 \mathrm{GHz}$. The CPU time for this simulation is 10 days, during which we approximately simulate $10 \mathrm{~min}$ of physical time until the quasiequilibrium state for the confined jet is reached.

\section{RESULTS AND DISCUSSIONS}

\section{Model validation}

To validate the LES results of the VFS-Geophysics model for the high Re jet flow, we simulated the experimental test case, which has a $R e$ number of $1.71 \times 10^{6}$. Let's call this test case the case O. In Fig. 2 we plot snapshots of simulated velocity magnitude on horizontal and vertical planes that pass through the center of the jet source at $t=8 \mathrm{~min}$ for the case $\mathrm{O}$. The simulation results in color maps of the jet velocity magnitude on the two planes can also be seen in the Movies 1 and 2 in the supplementary data. As shown in this figure the velocity magnitude decays at distances farther away from the jet source. In order to compare the computed and measured results for the velocity field, we time averaged the simulated results for the same period of time that measured data is collected $(=120 \mathrm{~s})$. To do so, we first continued the simulation until a quasi-equilibrium state is reached. This is checked by monitoring the computed instantaneous turbulent kinetic energy of the system, which leveled off after about $8 \mathrm{~min}$.

The time averaged contours of velocity magnitude along with velocity vectors for the vertical and horizontal planes of the jet are plotted in Fig. 3. As shown in this figure, the time averaged results shows that large secondary flows are present near the bed, side walls and on the free-surface. These secondary flow regions are also present in the experimental measurements. In Figs. 4 and 5 we present our measured and simulated results for the velocity magnitude on the horizontal and vertical planes that 
pass through the center of the jet source. Note that we, for the first time, are reporting an experimental and numerical caparison for such a high-Re confined jet. As shown in Figs. 4 and 5, great agreement between the measured and LES captured velocity magnitude can be seen.

\section{Sand-pile effect}

In order to further analyze the effect of the frozen sand-pile on the velocity distribution of the jet, we carried out an extra LES of the jet flow with the identical grid system, boundary conditions, and $R e$ number. The only difference in this simulation, which is called case A, comparing to the case $\mathrm{O}$ (described above) is that we numerically removed the sand pile and assumed that the rigid bottom of the rectangular flume is flat throughout the length of channel. The LES of this hypothetical case is carried out for the same duration of physical time as that of the former case and then time averaged. We note that the Re number of the two cases $\mathrm{O}$ and $\mathrm{A}$ are the same and the only difference between the two cases is the bed geometry of the flume.

In Figs. 6 and 7 we compared the measured data with the LES results with and without the frozen sand-pile. As one can readily see in these two figures, presence of the sand pile has no significant effect on the computed velocity profile up until about $7 D$ from the jet source. Only after about $8 D$ from the jet source the discrepancy between the two LES simulations becomes significant. At distance more than about $8 D$, the peak of velocity magnitude at the core of the jet is underestimated when the sand pile is removed from computations. The vertical distribution of velocity magnitude is more sensitive to the presence of the sand pile. This is evident in Fig. 7, where the dashed line (i.e. the LES results without sand pile) shows a more significant discrepancy in vertical velocity distribution than that of horizontal velocity in Fig. 6.

This finding has an important implication for the design of stilling basins where the excessive energy of an outlet jet, e.g. bottom outlet of dams and sluice gates, needs to be dissipated. In such a system, bed scour usually leads to formation of a sand pile similar to that we presented in this study. Our LES results show that presence of such sand pile downstream of a stilling basin has a negative effect on the dissipation of the high velocity at the core of the jet. This is evident in Figs. 6 and 7 (see the dashed lines) in which the presence of a frozen sand-pile has led to higher core velocity (see the dashed lines in Figs. 6 and 7).

\section{$R e$ number effect}

The simulated instantaneous quantities of the flow field at different points along the axes of jet source for the case $\mathrm{O}$ are recorded. These instantaneous values are used to obtain the power spectral density (PSD) of the streamwise velocity fluctuations. In Fig. 8 we plot the computed PSD of the jet flow in case $\mathrm{O}$ for which the $R e$ number is $1.71 \times 10^{6}$. Using the shown PSD one can identify the range of scales of the vortical structures that are resolved by the LES model. The region with -1 and $-5 / 3$ slopes in this figure represents the large-scale motions (i.e. production range at which the turbulent kinetic energy is produced) and inertial subrange (i.e. range of frequencies at which the cascade of energy takes place), respectively. Range of frequencies higher than the $-5 / 3$ slope region is know as the dissipation range, which indicates the dissipation of 
turbulent kinetic energy by subgrid scale motions due to molecular and SGS model viscosity. In other word, the eddies at the dissipation range are not directly resolved by the LES model because their size is smaller than the filter size (spatial step) of the LES in this work. Instead, their effect in dissipating the turbulence kinetic energy is modeled via SGS model.

Now let's focus our attention to the PSD data in Fig. 8. The size of the smallest eddies depends on the Re number. The higher the Re, the smaller is the size of smallest eddies (Davidson 2004). The local velocity and consequently Re number is higher near the jet source that those of farther distances. Thus, one expect that the smallest eddies at $x=5 D$ to be smaller than those at $x=15 D$. This can be seen in Fig. 8 where the range of scales that are not resolved by LES decreases with the distance from jet source. We note that the spatial step and thus the filter size throughout the simulation domain remains identical, but, the local Re number and therefore the eddy sizes vary. At $x=15 D$, a greater range of high frequency (i.e. small scale) motions are resolved than that of $x=5 D$.

In order to further analyze the $R e$ effect on the PSD of the streamwise velocity fluctuations. We simulated the case $\mathrm{O}$ with a bulk velocity that is reduced by 90 percent. All other characteristics including the grid system remain the same. In this case, i.e. the so-called case $\mathrm{B}$, the $R e$ number becomes equal to $1.71 \times 10^{5}$, which one order of magnitude smaller than that of case $\mathrm{O}$. The high frequency ranges for case $\mathrm{B}$ is expected to contain eddies that are larger in size that those of case $\mathrm{O}$. Thus, in case B, LES is expected to resolve a broader range of the scales. This can be seen in Fig. 9 where we plot the PSD of the streamwise velocity fluctuations at different distances from the jet source. Finally, it is noteworthy to mention that the overall turbulent kinetic energy of the system in case $\mathrm{O}$ is higher than that of the case $\mathrm{B}$. This is because the Re number of the former case is one order of magnitude higher than that of the latter case. The PSD data, shown in Fig. 10, clearly show the level of kinetic energy in the two test cases.

Finally, we note that the discussion provided in this section is merely focused on the effect of Re number on the frequency of eddies present in the flow. (Fellouah and Pollard 2009) showed that the length scale of eddies in the near to fully-developedturbulent-mixing region of a round-free-turbulent jet will follow the expression of $\iota / D \approx 0.04$, where $\iota \approx U_{l} / f_{l}, U_{l}$ is the local mean-velocity, $f_{l}$ is the local eddy frequency, $\iota$ is the local eddy size, and $D$ is the jet diameter at the jet exit. The $\iota / D$ ratio in Figs. 8 to 10 at frequencies of $10 \mathrm{~Hz}$ and above is about 0.76 meaning that the jet flow is not at its fully-developed region and that computations should have continued for longer distances downstream to get to the near-fully-developed region of the jet.

In summary, we present the experimentally measured velocity distribution downstream of a high-Re confined jet. The velocity distribution shows a monotonic decay of velocity due to a strong turbulent diffusion. The jet flow in this study was confined by free-surface, rigid bed, and vertical side wall. We also employed the LES module of the VFS-Geophysics model to simulate the jet flow we created in our experiment. The simulation results for the velocity magnitude on two perpendicular planes of the 
jet showed great agreement with the measured data. LES results for the two cases of with and without frozen sand-pile in the main channel revealed that: $(i)$ at distances less than $7 D$ from the jet source, the frozen sand-pile has no significant effect on the velocity profile of the jet in the channel; (ii) at distances farther than $7 D$ from the jet source, the presence of frozen sand-pile leads to higher velocity core for the jet than that of the case without sand-pile; and (iii) the presence of sand pile downstream of the jet impedes the decay of high velocity core of the jet. The latter conclusion has an important implication for the design of outlet works downstream of outflow jets into water bodies: in order to facilitate the mixing of the high-Re jet flows, one needs to minimize the deposition of sediment material in the outlet area.

Given the jet velocity $\left(U_{b}=6.29 \mathrm{~m} / \mathrm{s}\right)$ and depth $(1.09 \mathrm{~m})$ at the source (see Fig. $2(B))$, the jet creates a high-Froude-number $(F r=1.92)$ flow in which the gravity waves on the free-surface can be of significant size. Due to the high computational cost of free-surface calculations, these waves are not simulated in this work. However, we plan to further investigate this issue in a future study. Nevertheless, we acknowledge that the water-surface fluctuations observed in the laboratory are about $5 \mathrm{~cm}$. Thus, use of rigid-lid assumption for the free-surface is a major drawback of our LES simulations, however, the calculated pressure field at the free-surface adjust itself in a way that it compensates to some extend for the free-surface fluctuations occurring in physics. To show such numerical adjustments, we calculate the instantaneous freesurface elevation of the simulation results using the simulated pressure field, which is shown in Movie 3 in the supplementary data.

\section{ACKNOWLEDGMENTS}

This work was supported by a grant from Barr Engineering, Bloomington, Minnesota. For additional information regarding the data generated from these simulations the reader can contact Ali Khosronejad via Email: ali.khosronejad@stonybrook.edu.

\section{REFERENCES}

Albertson, M. L., Dai, Y. B., Jensen, R. A., and Rouse, H. (1950). "Diffusion of submerged jets." Transactions of the American Society of Civil Engineers, 115(1), 639664.

Bogey, C. and Bailly, C. (2009). "Turbulence and energy budget in a self-preserving round jet: direct evaluation using large eddy simulation." Journal of Fluid Mechanics, 627, 129-160.

Bogey, C., Marsden, O., and Bailly, C. (2012). "Influence of initial turbulence level on the flow and sound fields of a subsonic jet at a diameter-based reynolds number of 100,000.” Journal of Fluid Mechanics, 701, 352-385.

Borazjani, I., Ge, L., and Sotiropoulos, F. (2008). "Curvilinear immersed boundary method for simulating fluid structure interaction with complex 3D rigid bodies." Journal of Computational Physics, 227, 7587-7620.

Capp, S. P., Hussein, H. J., and Georgew, W. K. (1990). "Velocity measurements in a high reynolds number, momentum-conserving, axisymmetric, turbulent jet.." Report no., Turbulence Research Laboratory, University at Buffalo, SUNY. 
Chedevergne, F., Leon, O., Bodoc, V., and Caruana, D. (2015). "Experimental and numerical response of a high-reynolds-number $\mathrm{m}=0.6$ jet to a plasma synthetic jet actuator." International Journal of Heat and Fluid Flow, 56, 1-15.

Craft, T. and Launder, B. (2001). "On the spreading mechanism of the threedimensional turbulent wall jet." Journal of Fluid Mechanics, 435, 305-326.

Davidson, P. A. (2004). Turbulence. Oxford University Press.

Fellouah, H. and Pollard, A. (2009). "The velocity spectra and turbulence length scale distributions in the near to intermediate regions of a round free turbulent jet." Phys. Fluids, 21.

Ge, L. and Sotiropoulos, F. (2007). "A numerical method for solving the 3D unsteady incompressible navierstokes equations in curvilinear domains with complex immersed boundaries." Journal of Computational Physics, 225, 1782-1809.

Hall, J. W. and D., E. (2007). "Three-dimensional turbulent wall jets issuing from moderate-aspect-ratio rectangular channels." AIAA Journal, 45(6), 1177-1186.

Hussein, H. J., Capp, S. P., and George, W. K. (1994). "Velocity measurements in a high-reynoldsnumber, momentum-conserving, axisymmetric, turbulent jet." Journal of Fluid Mechanics, 258, 31-75.

Kang, S., Lightbody, A., Hill, C., and Sotiropoulos, F. (2011). "High-resolution numerical simulation of turbulence in natural waterways." Advances in Water Resources, 34(1), 98-113.

Khosronejad, A. and Rennie, C. (2010). "Three-dimensional numerical modeling of unconfined and confined wall-jet flow with two different turbulence models." Canadian Journal of Civil Engineering, 37, 576-587.

Khosronejad, A. and Sotiropoulos, F. (2014). "Numerical simulation of sand waves in a turbulent open channel flow." Journal of Fluid Mechanics, 753, 150-216.

Kwon, S. J. and Seo, I. W. (2005). "Reynolds number effects on the behavior of a non-buoyant round jet." Experiments in Fluids, 38, 801-812.

Mungal, M. G. and Hollingsworth, D. K. (1989). "Organized motion in a very high reynolds number jet." Physics of Fluids A, 1, 1615-1623.

Mungal, M. G., Lozano, A., and van Cruyningen, I. (1992). "Large-scale dynamics in high reynolds number jets and jet flames." Experiments in Fluids, 12, 141-150.

Panchapakesan, N. R. and Lumley, J. L. (1993). "Turbulence measurements in axisymmetric jets of air and helium. part 1. air jet." Journal of Fluid Mechanics, 246, 197-223.

Rajaratnam, N. (1976). Turbulent jets. Elsevier.

Rajaratnam, N. and Pani, B. (1974). "Three-dimensional turbulent wall jets." Journal of Hydraulic Division, 100, 69-83.

Rajaratnam, N. and Subramanya, K. (1967). "Flow immediately below submerged sluice gate." Proceedings of the Hydraulics Division, ASCE, HY4, 57-77.

Rodi, W. (1975). "A new method of analyzing hot-wire signals in highly turbulent flow and its evaluation in a round jet." DISA Information.

Wu, S. and Rajaratnam, N. (1995). "Free jumps, submerged jumps and wall jets." Journal of Hydraulic Research, 33(2), 197-212.

Wygnanski and Fiedlehr (1969). "Some measurements in the self-preserving jet." Jour- 
$316 \quad$ nal of Fluid Mechanics, 38, 577-612.

317 Yoda, M., Hesselink, L., and Mungal, M. G. (1992). "The evolution and nature of $318 \quad$ large-scale structures in the turbulent jet." Physics of Fluids A. 
TABLE 1. Experimental set up of the jet in the main channel of SAFL. $Q$ is the discharge of the jet and the bulk velocity at the jet source $\left(U_{b}\right)$ is equal to $4\left(\pi D^{2}\right)^{-1} Q$.

\begin{tabular}{llllc}
\hline \hline$Q\left(m^{3} s^{-1}\right)$ & $\nu\left(m^{2} s^{-1}\right)$ & $D(m)$ & $U_{b}\left(m s^{-1}\right)$ & $R e$ \\
\hline 0.4587 & $1.12^{-6}$ & 0.3048 & 6.29 & $1.71 \times 10^{6}$ \\
\hline \hline
\end{tabular}

TABLE 2. Geometry of sand pile relative to the origin, i.e. point 1 in Fig. $1(C)$. The schematic location of points 2 to 7 , which represent the frozen sand-pile, can also be seen in Fig. 1 (C)). We note that the geometry of the frozen sand-pile is uniform in spanwise $(y)$ direction.

\begin{tabular}{lllccccc}
\hline \hline & Jet source & point 2 & 3 & 4 & 5 & 6 & 7 \\
\hline$x(m)$ & 0 & 8.03 & 8.53 & 9.14 & 9.75 & 10.36 & 10.97 \\
$z(m)$ & 0 & 0.17 & 0.25 & 0.41 & 0.71 & 0.86 & 0.86 \\
\hline \hline
\end{tabular}

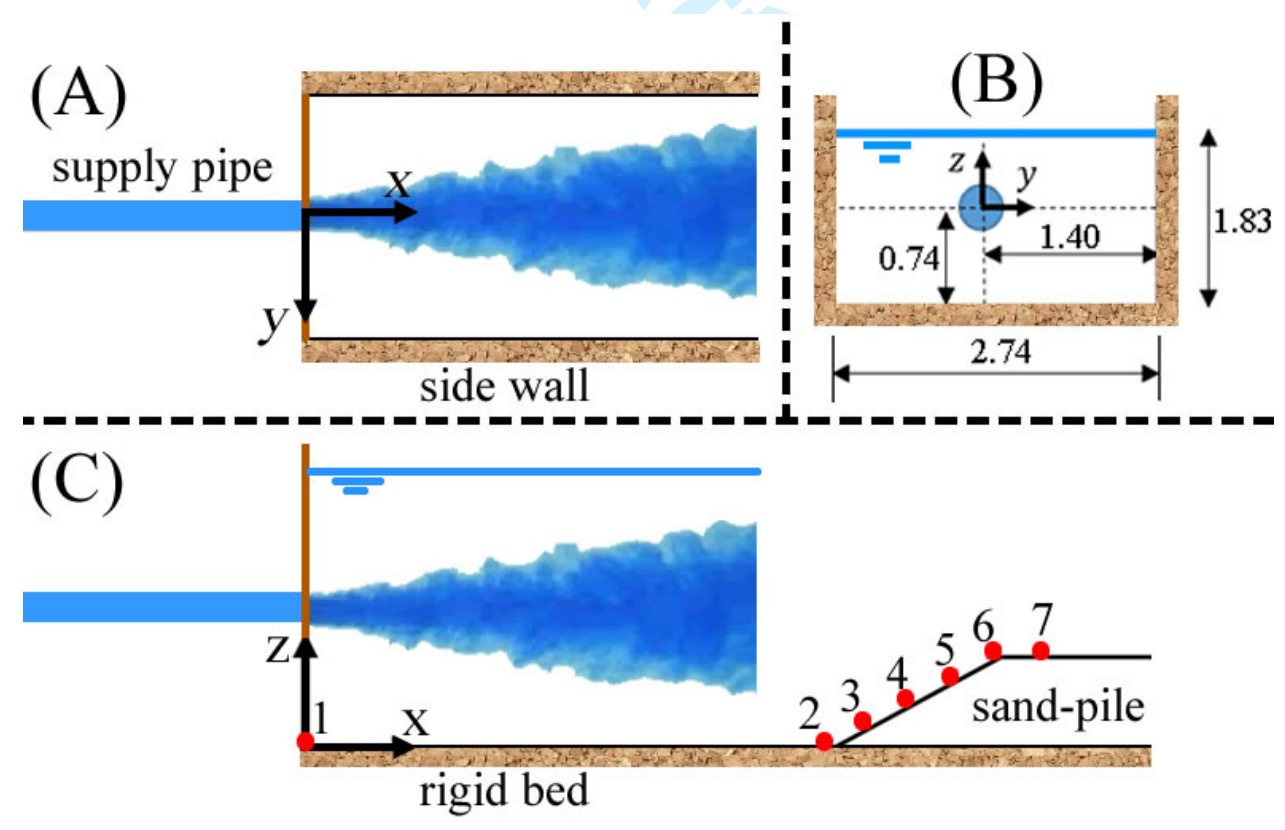

FIG. 1. Schematic of the experimental setup from (A) plan and (B) crosssectional, and (C) side views. Dimensions are in $m$ and jet flow is from left to right. 

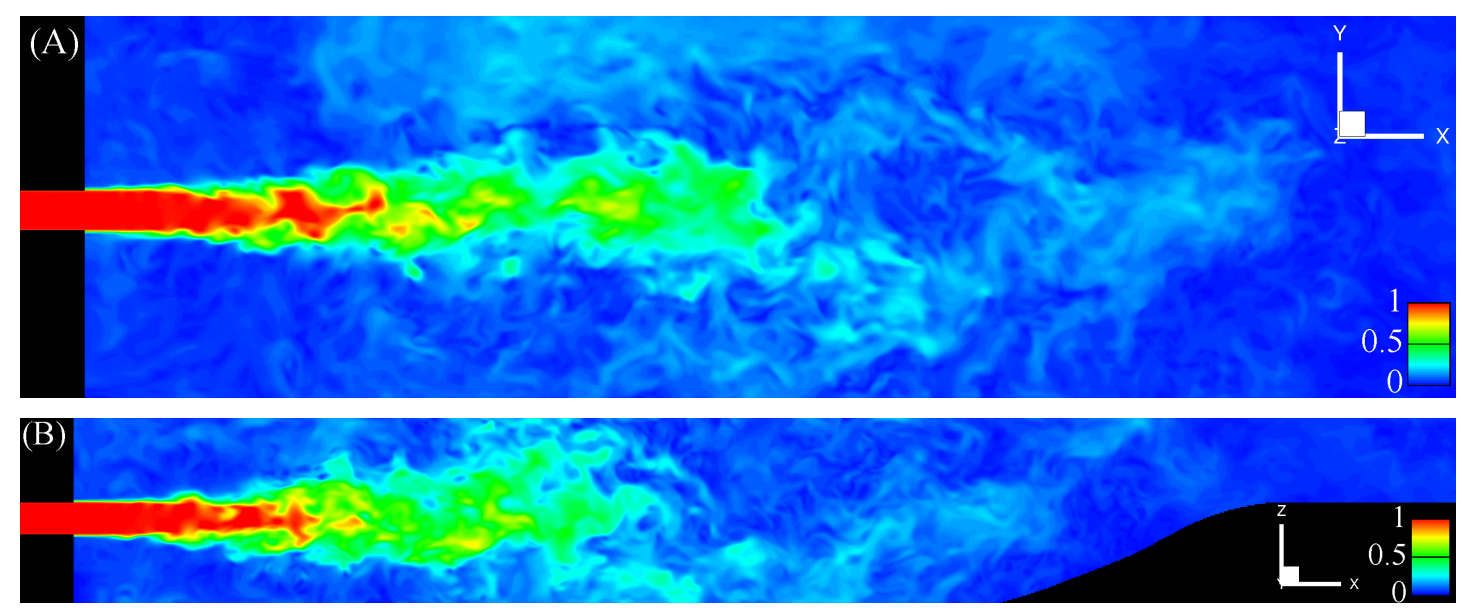

FIG. 2. Simulated instantaneous contours of velocity magnitude at $t=$ $8 \min$ on (A) horizontal and (B) vertical planes that pass through the center of the jet source. The black area at the end of the domain in (A) shows the frozen sand-pile. Velocity magnitude is non-dimensionalized with $U_{b}$. Flow is from left to right.
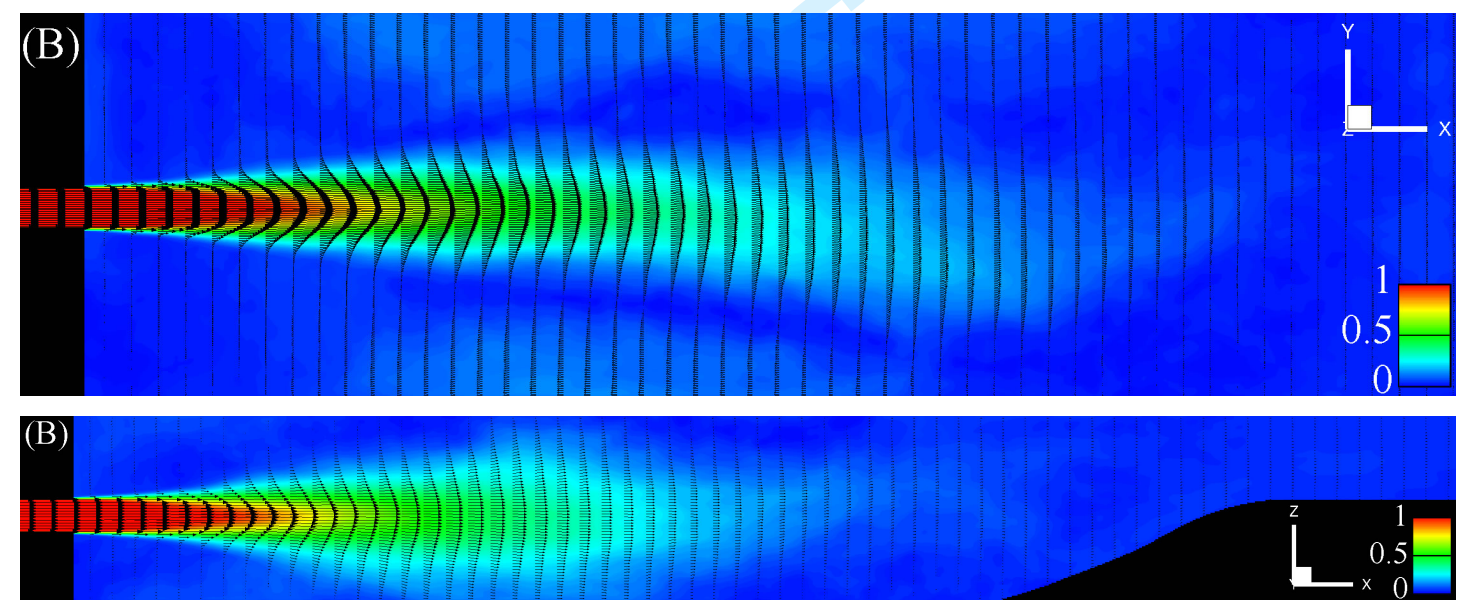

FIG. 3. Simulated time averaged velocity vectors and contours of velocity magnitude on (A) horizontal and $(B)$ vertical planes that pass through the center of the jet source. The black area at the end of the domain in (A) shows the frozen sand-pile. Velocity magnitude is non-dimensionalized with $U_{b}$. Velocity vectors in streamwise $(x)$ direction are skipped by a factor of 10. Flow is from left to right. 

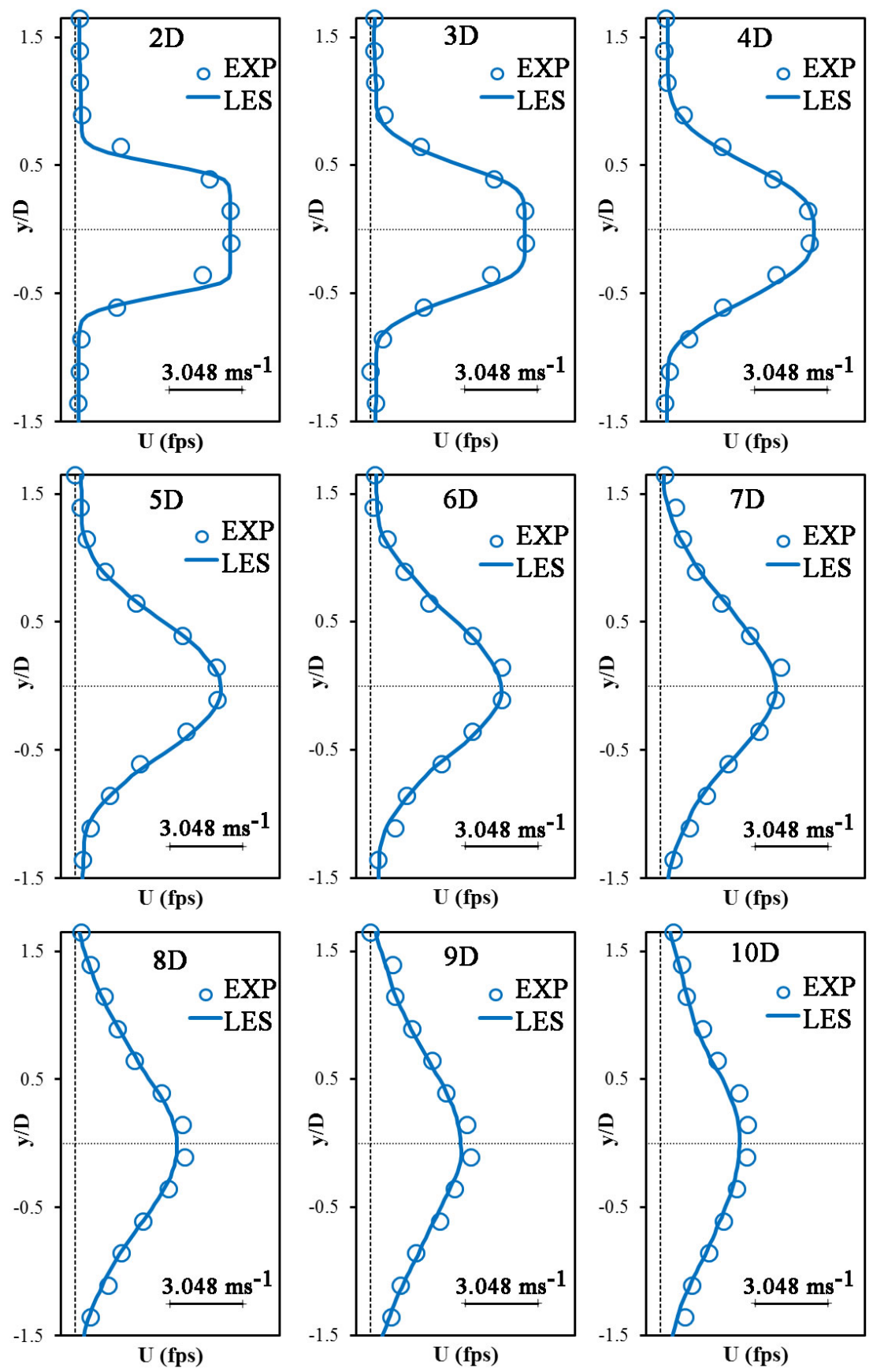

FIG. 4. Measured (circles) and LES (solid line) velocity magnitude $(U)$ at different distances (i.e. $x=2 D$ to $10 D$ ) from the jet source on a horizontal plane, which passes through the centroid of the jet source. $y$ is the spanwise coordinate that is non-dimensionlized with $D$. The jet source's center is located at $y / D=0$ and negative values of $y / D$ correspond to the left of the centroid. 

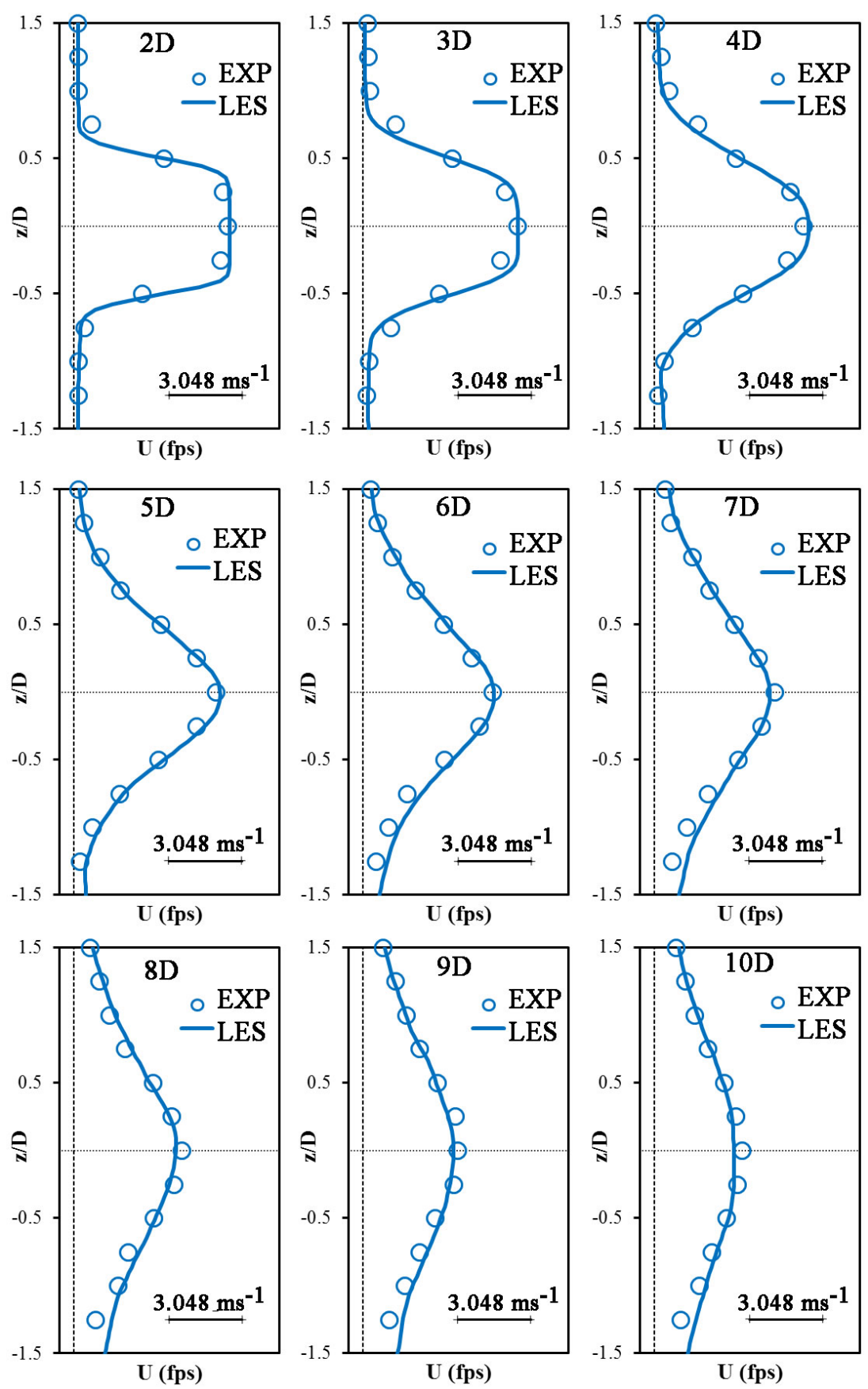

FIG. 5. Measured (circles) and LES (solid line) velocity magnitude $(U)$ at different distances (i.e. $x=2 D$ to $10 D$ ) from the jet source on a vertical plane, which passes through its centroid. $z$ is the vertical coordinate direction non-dimensionlized with $D$. Centroid of the jet source is located at $z / D=0$ and negative values of $z / D$ correspond to the elevations below it. 

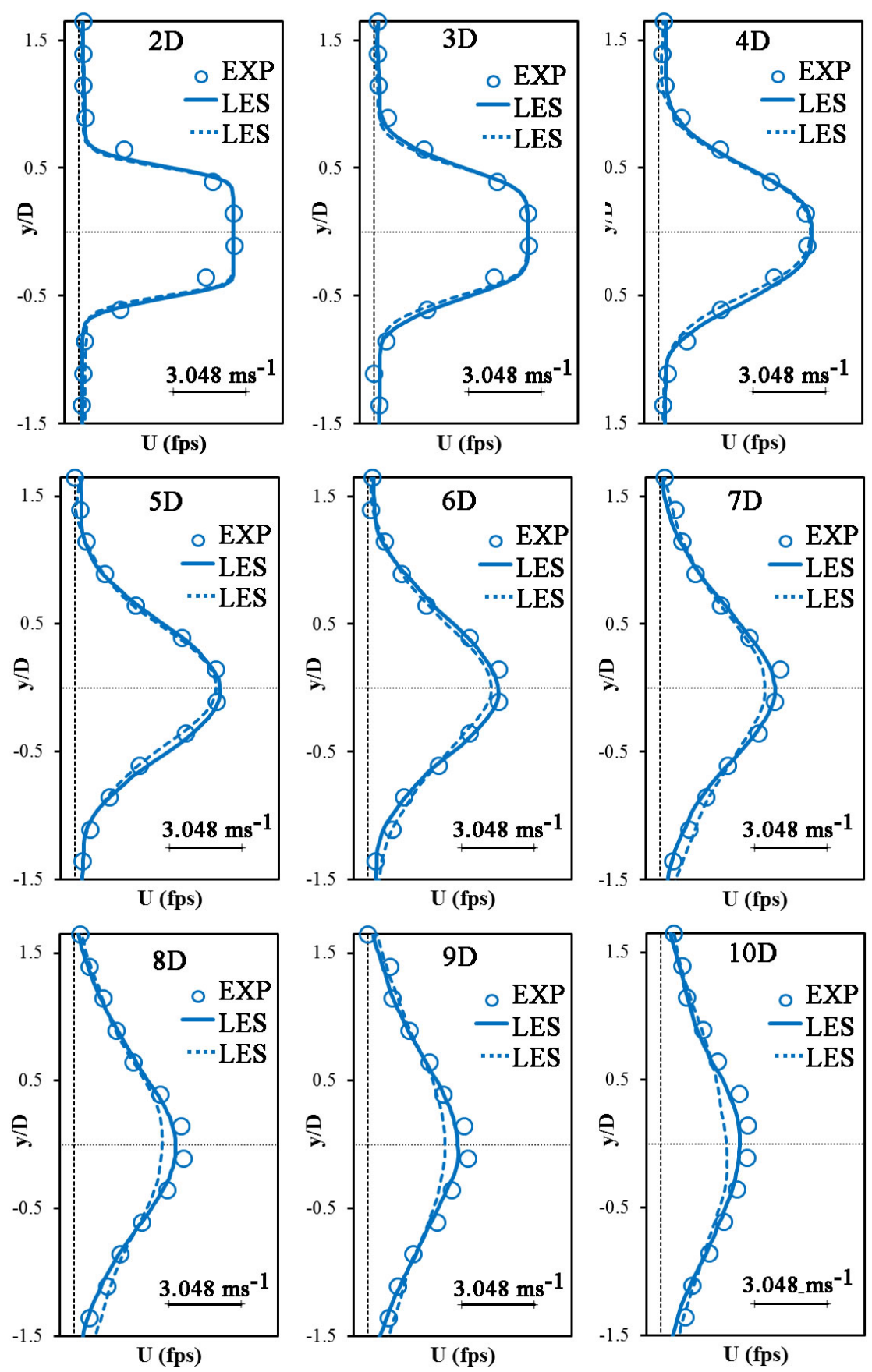

FIG. 6. Measured (circles) and computed (solid line) velocity magnitude $(U)$ at different distances (i.e. $x=2 D$ to $10 D$ ) from the jet source on a horizontal plane, which passes through its centroid. Dashed line shows the LES simulated results for a hypothetical case in which the sand pile is removed. 

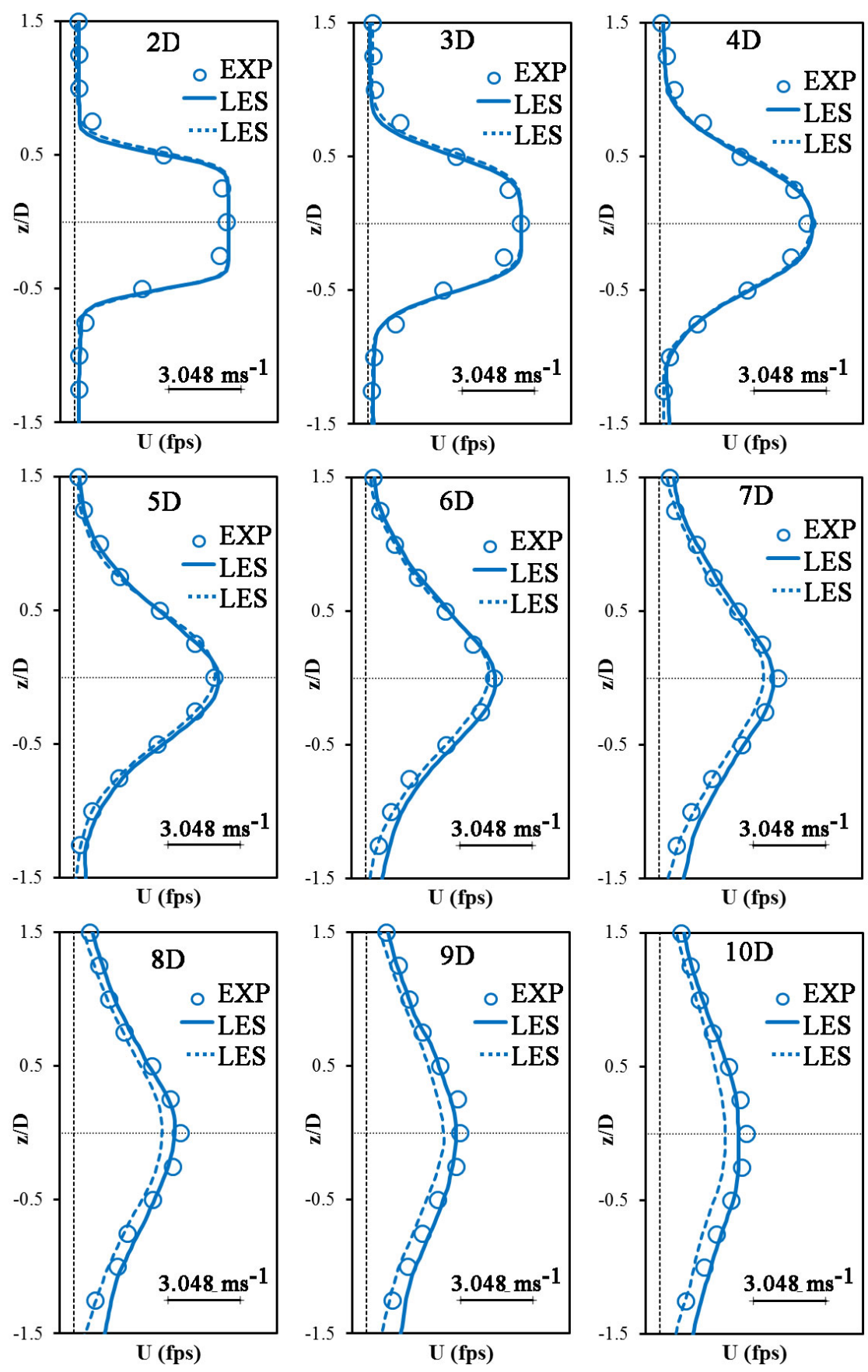

FIG. 7. Measured (circles) and computed (solid line) velocity magnitude $(U)$ at different distances (i.e. $x=2 D$ to $10 D$ ) from the jet source on a vertical plane, which passes through the center of its centroid. Dashed line shows the LES simulated results for a hypothetical case in which the sand pile is removed. 


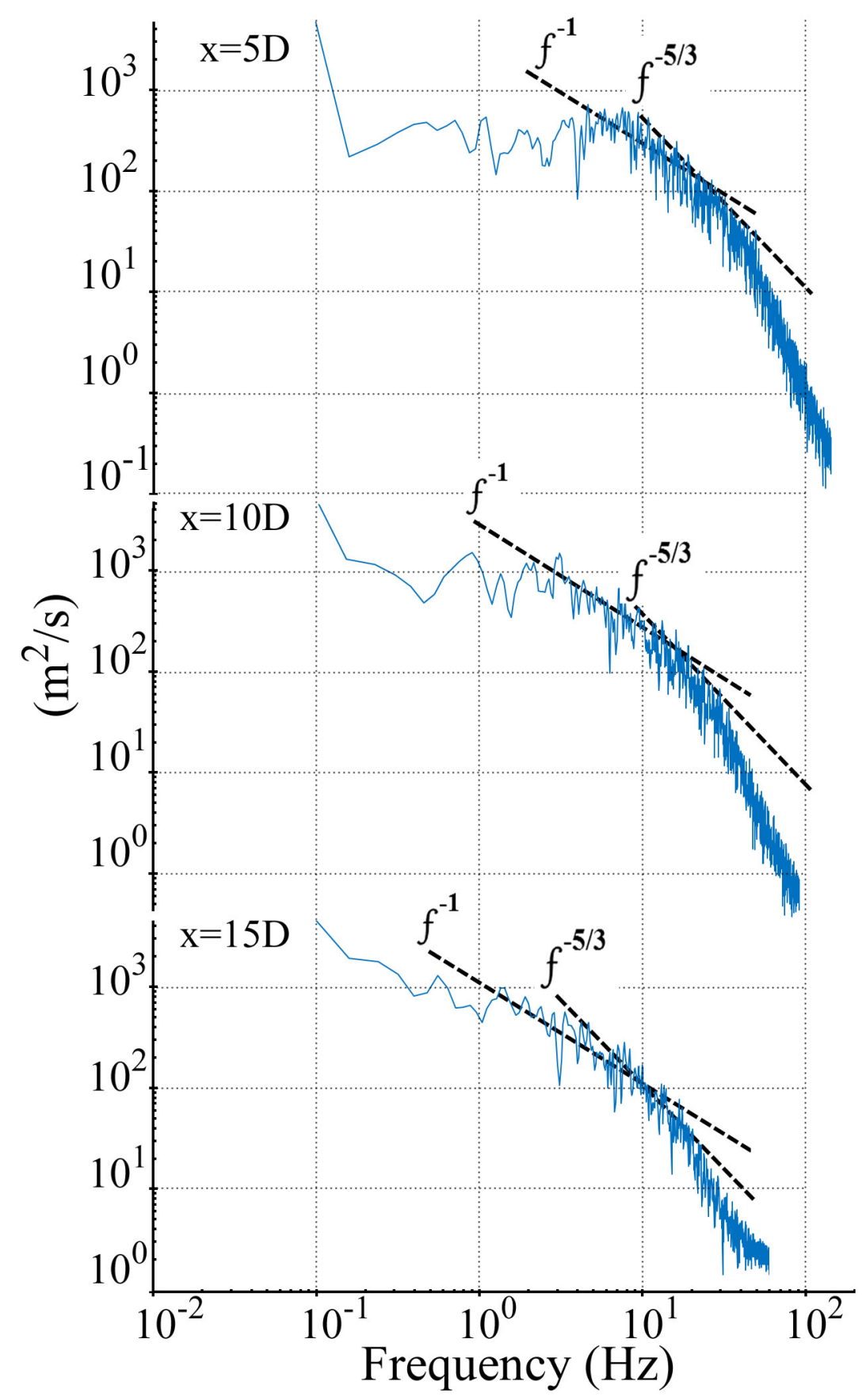

FIG. 8. Computed PSD of the streamwise velocity fluctuations in case 0 at different distance from jet source along the axes of the jet. 


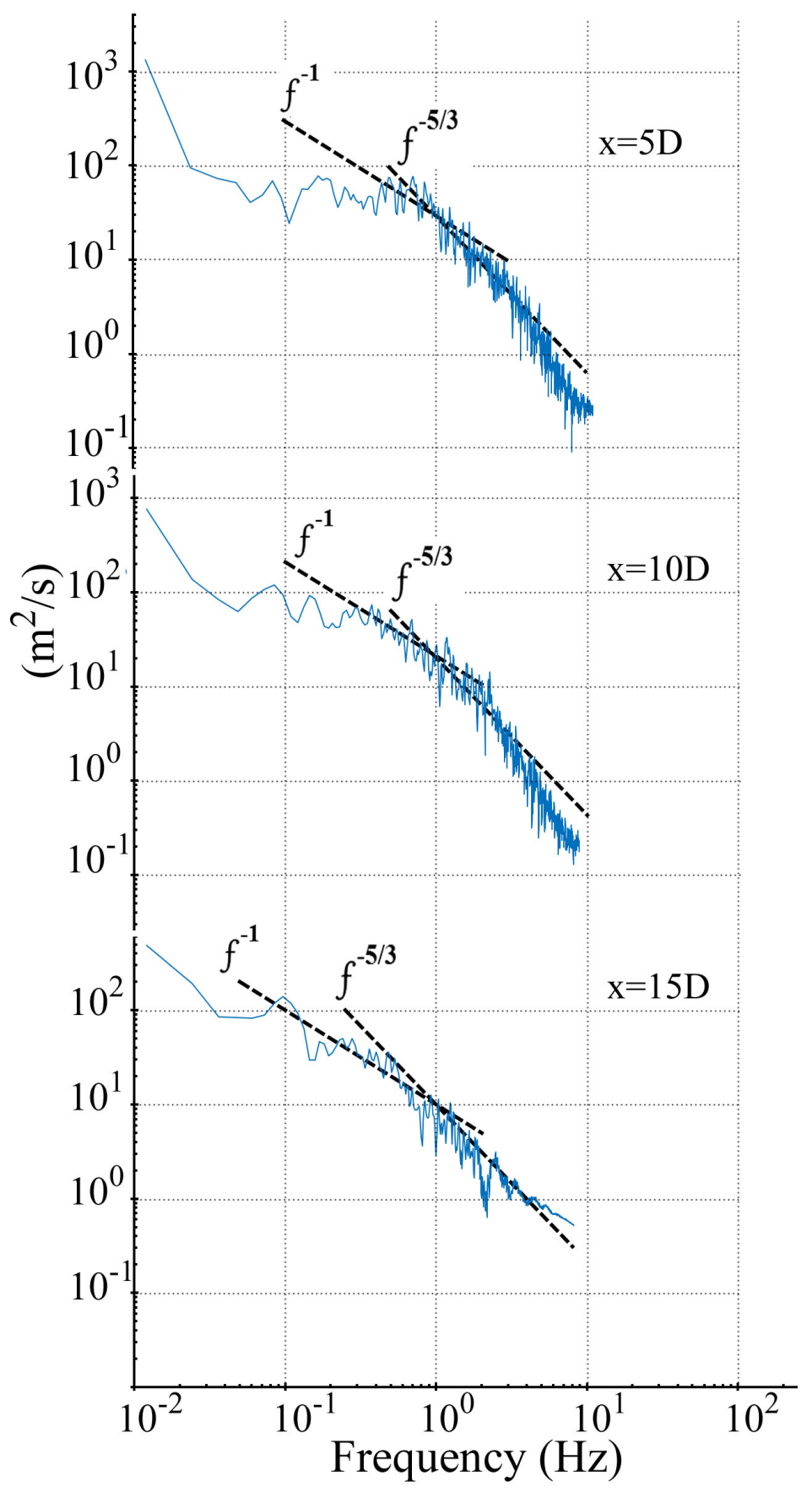

FIG. 9. Computed PSD of the streamwise velocity fluctuations in case B at different distance from jet source along the axes of the jet. 


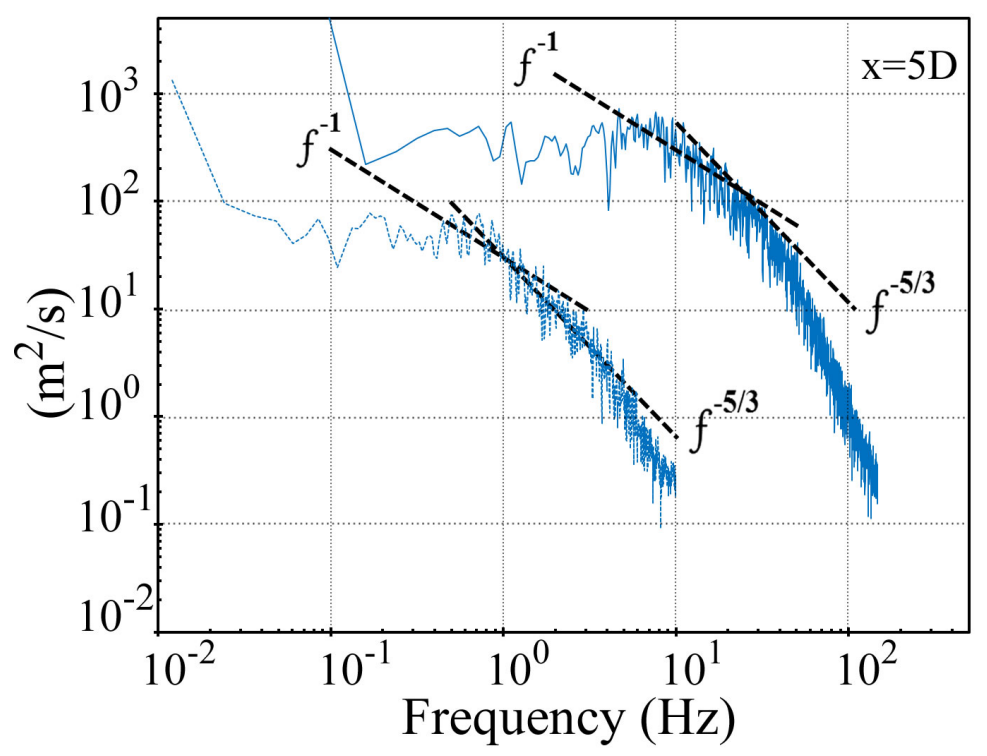

FIG. 10. Computed PSD of the streamwise velocity fluctuations in case 0 (solid blue line) and $\mathbf{B}$ (dashed blue line) at $x=5 D$ from jet source along the axes of the jet. 